\title{
The Kaon Bag Parameter at Physical Mass
}

\author{
Julien Frison*1, Peter Boyle ${ }^{1}$, Norman H. Christ ${ }^{2}$, Nicolas Garron ${ }^{3}$, \\ Robert Mawhinney ${ }^{2}$, Chris T. Sachrajda ${ }^{4}$, Hantao Yin ${ }^{2}$ \\ School of Physics \& Astronomy, University of Edinburgh, EH9 3JZ, UK ${ }^{1}$, \\ Physics Department, Columbia University, New York, NY 10027, USA ${ }^{2}$, \\ School of Mathematics, Trinity College, Dublin, Ireland ${ }^{3}$, \\ School of Physics and Astronomy, University of Southampton, SO17 1BJ, UK \\ E-mail: jfrison@ph.ed.ac.uk, paboyle@ph.ed.ac.uk, \\ nhc@phys.columbia.edu, ngarron@maths.tcd.ie, \\ rdmephysics.columbia.edu, ctsesoton.ac.uk, \\ yinnht@phys.columbia.edu
}

\section{RBC-UKQCD Collaboration}

We present preliminary results for the calculation of the Kaon Bag parameter $B_{K}$ in $N_{f}=2+1$ lattice QCD, using Möbius Domain Wall Fermion ensembles generated by the RBC-UKQCD collaboration. This computation is done directly at physical meson masses, so that we do not have to rely on chiral perturbation theory or any other mass extrapolation. In parallel, the four-quark operator is renormalised through the Rome-Southampton technique. Finally, we compare our value with previous results and draw some conclusions about the remaining dominant contributions in our error budget.

31st International Symposium on Lattice Field Theory - LATTICE 2013

July 29 - August 3, 2013

Mainz, Germany

${ }^{*}$ Speaker. 


\section{Introduction}

The mixing of neutral mesons such as the Kaon plays an important role in $C P$-violation, as the so-called box-diagrams involve terms like $V_{x s}^{*} V_{x d}$. However, those perturbative diagrams cannot deal with the confinement of ingoing and outgoing quarks. Nevertheless, an Operator Product Expansion (OPE) allows a separation between the perturbative part and a non-perturbative QCD part that can be computed on the lattice. Lattice computation have already been very successful on this path (see [1,2] for a review, and [3,4] for previous results of our collaboration), and what is at stake is now an improvement of the uncertainties.

In the context of lattice computation, we traditionally express the $K^{0}-\bar{K}^{0}$ matrix element as a ratio normalised by the result of Vacuum Saturation Approximation. On a practical side we therefore have to compute three-point and two-point functions, and fit a ratio on ranges that form a plateau converging to the asymptotic constant.

Then, this result has to be brought to a renormalisation scheme which is common with the perturbative computations. This has been done through the Rome-Southampton method and using several intermediate schemes to evaluate the systematic error coming from this part of the computation. In order to improve the convergence to the perturbative regime, non-exceptional momenta have been used and the matching is done at a scale of $3 \mathrm{GeV}$.

The present calculation, which is preliminary, has been done on the new $N_{f}=2+1$ Möbius Domain-Wall Fermion (MDWF) ensembles of the RBC-UKQCD collaboration, generated directly at the physical pion mass. We used two ensembles with roughly the same physical volume $\left(M_{\pi} L \simeq\right.$ 3.8) but different lattice spacing: a $48^{3} \times 96$ ensemble at $\beta=2.13$ and a $64^{3} \times 128$ ensemble at $\beta=2.25$. Finite-volume errors are estimated from older ensembles and are not a crucial issue. The bare $B_{K}$ ratios have been computed with respectively 60 and 21 configurations of those ensembles, while 11 and 6 configurations were sufficient for the Non-Perturbative Renormalisation (NPR) of these preliminary results. All of these measurements will ultimately be performed on larger data sets as part of a forthcoming publication, and as such this work is prelimary.

\section{Bare $B_{K}$ ratio}

For the bare ratio, all contractions have been computed using Coulomb gauge fixed wall sources and the All Mode Averaging (AMA) method. The latest combines propagators computed with different precisions (here $10^{-4}$ and $10^{-8}$ ) in order to improve signals at a minimal cost [5].

The ratio

$$
B_{K}^{\text {bare }}(t, \Delta t)=\frac{\left\langle K^{0}(\Delta t)\left|\mathscr{O}_{V V+A A}^{\Delta S=2}(t)\right| \bar{K}^{0}(0)\right\rangle_{A M A}}{\frac{8}{3}\left\langle K^{0}(\Delta t-t) \mid A_{0}(0)\right\rangle_{A M A}\left\langle A_{0}(t) \mid \bar{K}^{0}(0)\right\rangle_{A M A}}
$$

is fitted to a constant on the two-dimensional space $(t, \Delta t)$, The fit domain is chosen as $\Delta t \in$ $\left[\Delta t_{\text {min }}, \Delta t_{\text {max }}\right]$ and $t \in\left[t_{\text {margin }}, \Delta t-t_{\text {margin }}\right]$ (plus symmetric times).

For the $48^{3}$ ensemble we choose the domain $\left(t_{\operatorname{margin}}, \Delta t_{\min }, \Delta t_{\max }\right)=(8,20,32)$, giving

$$
B_{K}^{48}=0.5840(9)
$$

for an uncorrelated fit with $\chi^{2} / d o f=0.76$, while still giving an acceptable correlated $\chi^{2} / d o f=$ 1.68. On the $64^{3}$ ensemble stabilising the correlated fit becomes impossible and we perform an 
uncorrelated fit on the domain $\left(t_{\operatorname{margin}}, \Delta t_{\min }, \Delta t_{\max }\right)=(10,25,40)$, giving (with $\chi^{2} /$ dof $=0.64$, see Fig. 1)

$$
B_{K}^{64}=0.5627(7)
$$

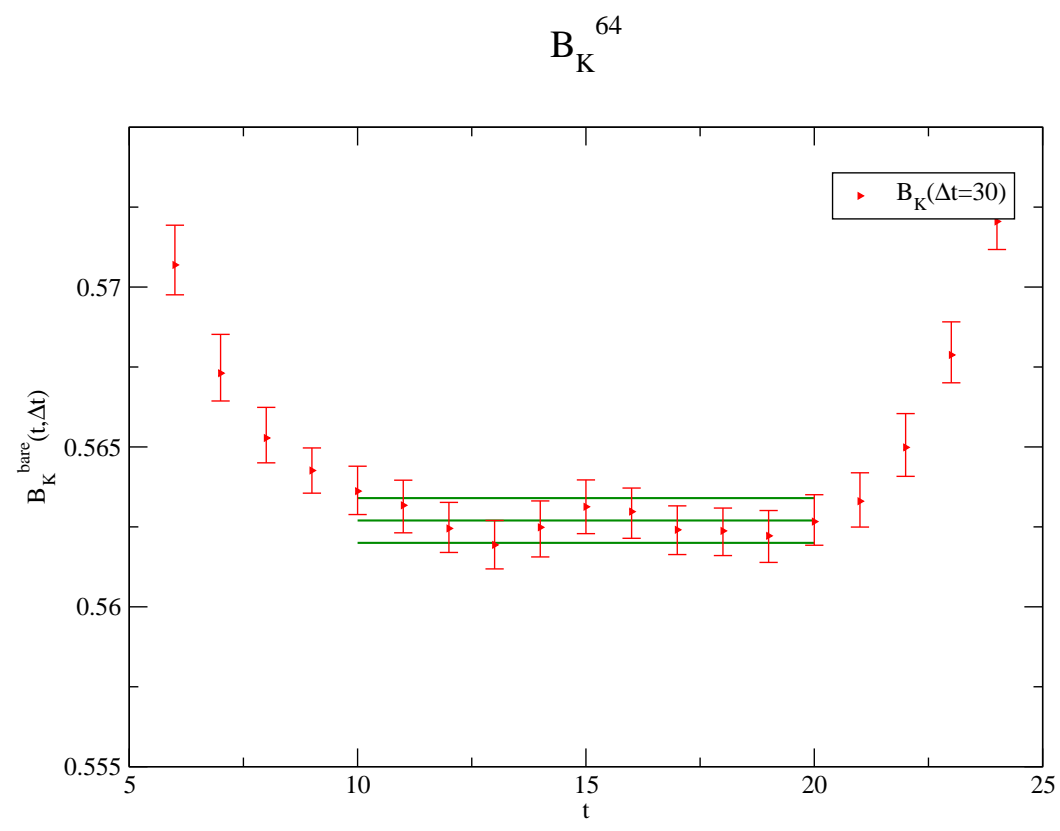

Figure 1: We show a part of the fit on the $64^{3}$ ensemble. Green lines represent the result of the fit with its error bars. The fit is actually two-dimensional and we only represent one $\Delta t$ timeslice for readibility.

\section{Non Perturbative Renormalisation}

We have to renormalise a four-quark operator, which hopefully renormalise multiplicatively in a Domain-Wall discretisation. In the RI/SMOM scheme this is done through the computation of four-quark Green's functions such as Fig. 2, where ingoing and outgoing quarks use Landau gauge-fixed wall sources with momenta on those walls.

We note $\Gamma_{V V+A A}$ the associated amputated Green's function. The scheme is completely defined once we choose a projector $P$ allowing us to write the renormalisation condition

$$
P \Gamma_{V V+A A}\left(\mathscr{O}_{V V+A A}^{R I}\right)=\left.P \Gamma_{V V+A A}\left(\mathscr{O}_{V V+A A}\right)\right|_{\text {tree }} .
$$

We will use the two choices

$$
\begin{aligned}
& P_{\mu}^{\gamma}=\delta_{i j} \delta_{k l}\left[\left(\gamma_{\mu}\right)_{\beta \alpha}\left(\gamma_{\mu}\right)_{\delta \gamma}+\left(\gamma_{\mu} \gamma_{5}\right)_{\beta \alpha}\left(\gamma_{\mu} \gamma_{5}\right)_{\delta \gamma}\right] \\
& P^{\phi}=\delta_{i j} \delta_{k l}\left[(q)_{\beta \alpha}(q)_{\delta \gamma}+\left(q \gamma_{5}\right)_{\beta \alpha}\left(q \gamma_{5}\right)_{\delta \gamma}\right] / q^{2}
\end{aligned}
$$

defining respectively the schemes $\mathrm{RI} / \mathrm{SMOM}_{\gamma_{\mu}}$ and $\mathrm{RI} / \mathrm{SMOM}_{\phi}$, where $i, j, k, l$ contract with the colour indices of $\Gamma_{V V+A A}$ and $\alpha, \beta, \gamma, \delta$ with its spin indices. 

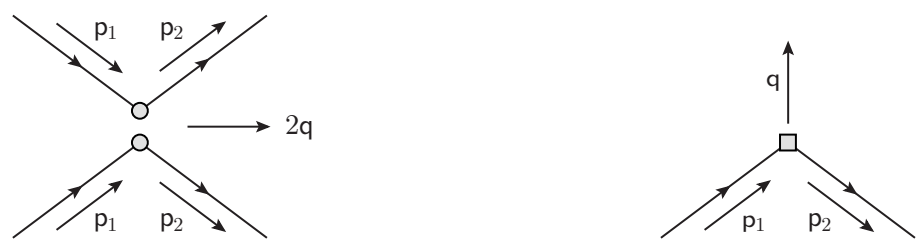

Figure 2: Four-quark Green's functions $G_{V V+A A}$ with momenta defining the RI/SMOM non-exceptional scheme (left), and the scheme definition for the bilinears (right). The momenta are chosen such that $p_{1}^{2}=$ $p_{2}^{2}=q^{2}$, where $q=p_{1}-p_{2}$. An additional momentum of $2 q$ (resp. $q$ ) leaves the operator as indicated by the arrow. Every momentum or subset of momenta is of order $q$ (never zero), which improves the pertubative running.

\begin{tabular}{|c|c|c|c|}
\hline$\beta$ & scheme & This work $\left(48^{3}\right.$ and $\left.64^{3}\right)$ & Previous results $\left(24^{3}\right.$ and $\left.32^{3}\right)$ \\
\hline 2.13 & $\overline{\mathrm{MS}}[\mathrm{NDR}](\gamma, 3 \mathrm{GeV})$ & $0.91764(4)$ & $0.91983(10)(51)(180)(3)$ \\
2.13 & $\overline{\mathrm{MS}}[\mathrm{NDR}](q, 3 \mathrm{GeV})$ & $0.94611(3)$ & $0.94672(11)(84)(63)(12)$ \\
2.25 & $\overline{\mathrm{MS}}[\mathrm{NDR}](\gamma, 3 \mathrm{GeV})$ & $0.94346(5)$ & $0.94284(17)(49)(48)(2)$ \\
2.25 & $\overline{\mathrm{MS}}[\mathrm{NDR}](q, 3 \mathrm{GeV})$ & $0.96722(2)$ & $0.96698(13)(86)(9)(2)$ \\
\hline
\end{tabular}

Table 1: $Z_{B_{K}}$ results from different ensembles and schemes. On the right column we remind previous results with a very similar action[3], where here the first number is statistical error, then scale setting error, then $m_{S}$ errors, then $Z_{A}-Z_{V}$ errors. Those number agree within systematic errors, and the statistical error has been drastically reduced even with a small number of configurations.

This condition gives us the factor

$$
\frac{Z_{V V+A A}}{Z_{q}^{2}}=\frac{\left.P \Gamma_{V V+A A}\left(\mathscr{O}_{V V+A A}\right)\right|_{\text {tree }}}{P \Gamma_{V V+A A}\left(\mathscr{O}_{V V+A A}^{R I}\right)}
$$

and we do the same for bilinear operators to get

$$
\frac{Z_{A}}{Z_{q}}=\frac{\left.P \Gamma_{A}\left(\mathscr{O}_{A}\right)\right|_{\text {tree }}}{P \Gamma_{A}\left(\mathscr{O}_{A}^{R I}\right)}
$$

so that $Z_{q}$ cancels in the ratio

$$
Z_{B_{K}}=\frac{Z_{V V+A A}}{Z_{A}^{2}}
$$

Those renormalisation factors, computed on the momenta $[p, 0, p, 0]$ with $p=9.25$ and $p=9.5$ thanks to boundary twisting, are run and matched to the pertubatively-defined $\overline{\mathrm{MS}}[\mathrm{NDR}]$ at scale $3 \mathrm{GeV}$, as shown in Fig. 3 with other results summarized in Tab. 1.

\section{Preliminary Continuum Extrapolation}

After multiplying our bare $B_{K}$ by those values for the two schemes $(\gamma, \gamma)$ and $(\not q, q)$, we can 


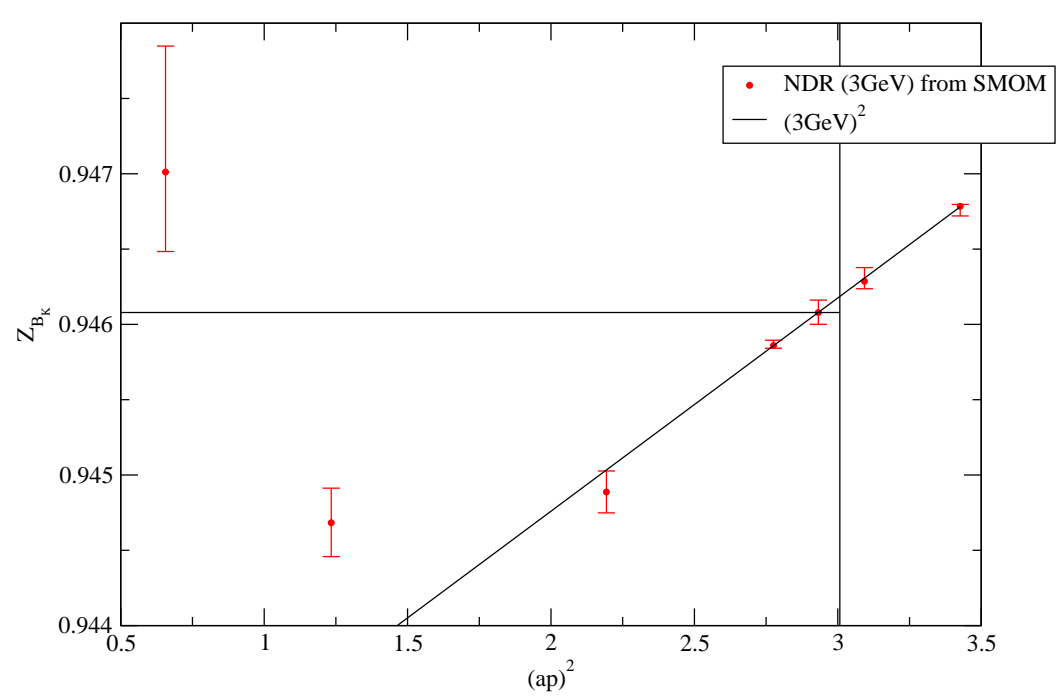

Figure 3: Renormalisation factor $Z_{B_{K}}$ in $\overline{\mathrm{MS}}[\mathrm{NDR}](3 \mathrm{GeV})$ from intermediate scheme RI/SMOM ${ }_{\phi}$, with $48^{3}$ ensemble. The vertical line shows the $(a p)^{2}$ value corresponding to the scale $|p|=3 \mathrm{GeV}$, while the horizontal line show the value for the closest simulated point relative to this scale. The slope of the linear fit is one of the ways to evaluate the systematic error due to not having an infinitely large Rome-Southampton window, although we prefer using the difference between different intermediate schemes at the end of the computation. Only the two points close to $3 \mathrm{GeV}$ are actually used, the other momenta having very low statistics.

extrapolate them to the continuum, removing $\mathscr{O}\left(a^{2}\right)$ effects :

$$
\begin{aligned}
& B_{K}^{\gamma, \gamma}=0.5241(11)_{\text {stat } 48}(17)_{\text {stat } 64}(4)_{a 48}(7)_{a 64} \\
& B_{K}^{q, q}=0.5332(12)_{\text {stat } 48}(17)_{\text {stat } 64}(7)_{a 48}(12)_{a 64},
\end{aligned}
$$

where we have separated errors coming from first the $B_{K}$ value on the $48^{3}$ lattice, then on the $64^{2}$ lattice, and then the errors propagated from each scale setting. The difference between those two values is an estimate of the systematic error due to the perturbation conversion from intermediate MOM schemes to $\overline{\mathrm{MS}}$. Concerning the central value any combination of these figures could theoretically be justified, however we choose to use the $(\not q, q)$ scheme as the reference as we observe a better perturbative matching from it. Including the estimation of finite-volume errors from our earlier ensembles ${ }^{1}$, we then quote the following preliminary result:

$$
B_{K}^{\overline{\mathrm{MS}}}(3 \mathrm{GeV})=0.533(3)_{\text {stat }}(0)_{\chi}(3)_{F V}(11)_{N P R}
$$

\section{Conclusion}

This value has to be compared to the previous results of our collaboration in [4]

$$
B_{K}^{\overline{\mathrm{MS}}}(3 \mathrm{GeV})=0.535(8)_{\text {stat }}(7)_{\chi}(3)_{F V}(11)_{N P R}
$$

\footnotetext{
${ }^{1}$ This is a conservative estimation, since the application of ChPT[6] suggests that this error is now much reduced.
} 


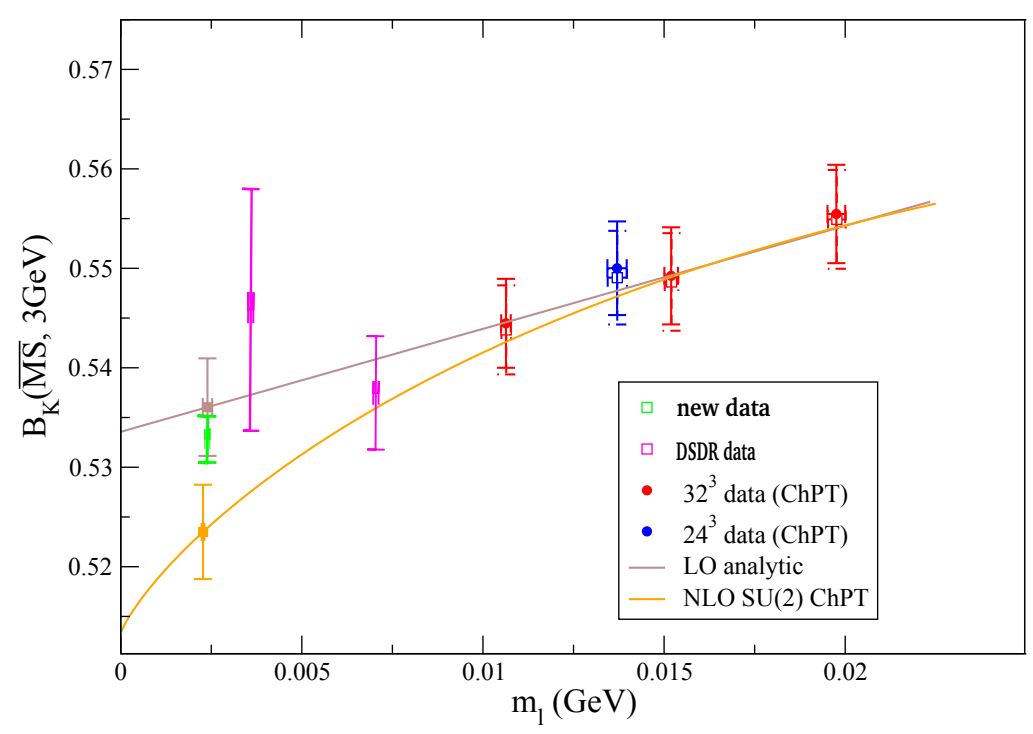

Figure 4: We present the results of this study, in green, compared to previous results used in a chiral extrapolation. The fits we show are only based on blue and red points, so that the systematic error separating the two formulae is very visible, although the purple data already suppressed much of this problem[4]. In the present study, on top of improving the statistical error, those systematics are completely removed.

\begin{tabular}{|c|c|}
\hline Collaboration & $\hat{B}_{K}$ \\
\hline \hline This work (preliminary) & $0.755(4)(15)$ \\
\hline RBC-UKQCD'12 & $0.758(11)(19)$ \\
RBC-UKQCD'10 & $0.749(7)(26)$ \\
BMW'11 & $0.773(8)(9)$ \\
SWME'11 & $0.716(10)(35)$ \\
Aubin'09 & $0.724(8)(29)$ \\
ETM'10 $\left(N_{f}=2\right)$ & $0.729(25)(17)$ \\
\hline
\end{tabular}

Table 2: RGI value for $B_{K}$ derived from different previous works. Other values might be found in $[1,2]$.

and the older [3]

$$
B_{K}^{\overline{\mathrm{MS}}}(3 \mathrm{GeV})=0.529(5)_{\text {stat }}(15)_{\chi}(2)_{F V}(11)_{N P R} .
$$

Although those result are perfectly compatible within error bars, we saw a slight increase of the central value with the inclusion of the near-physical mass ensembles, which reduced the gap between Taylor and ChPT fits and discarded large chiral logarithmic effects. The current analysis clearly confirms this phenomenum seen in [4].

We also provide a value in the Renormalisation Group Independent (RGI) scheme in Tab. 2 in order to allow for a comparison with results of some other collaborations.

We note that most determinations have a central slightly lower than the value we present. We suspect this could be related to ChPT fits outside their range of validity, and also the use of only $Z$ 
factors from schemes whose running is less well described by perturbation theory than RI/SMOM .

Most of the works also are, like this one, dominated by systematic errors. In our case, now that data down to the physical pion mass has eliminated the main systematic error, mass extrapolation, nearly all of the error is due to NPR.

Therefore we have shown that our new data and techniques provide an important improvement of the $B_{K}$ value, leading to $B_{K}^{\overline{\mathrm{MS}}}(3 \mathrm{GeV})=0.533(3)(11)$. This is still preliminary, and in particular would need more statistics on NPR to be confident of the currently negligible statistical error. The direct simulation at physical pion mass has been a very important ingredient, eliminating the dominant systematic error. The very obvious conclusion is now that what is important is improving the non-perturbative renormalisation. This would in particular imply determining $Z_{B_{K}}$ at higher energy scales, which in turn would imply simulating on finer and finer lattices. Renormalisation on finer lattices could be achieved on small volumes with step scaling. This includes running over a charm threshold since it is important to match to perturbation theory at scales above those where the real world charm becomes relevant. This will be a major objective for the next simulations. Additionally, the determination of $Z$ factors can also be improved by designing schemes with a better matching, a direction that has already been intensively studied by our collaboration, with improvements of the RI-MOM scheme already used in this work.

\section{Acknowledgements}

The authors gratefully acknowledge computing time granted through the STFC funded DiRAC facility (grants ST/K005790/1, ST/K005804/1, ST/K000411/1, ST/H008845/1). PAB acknowledges support from STFC Grant ST/J000329/1 and was also supported by the European Union under the Grant Agreement number 238353 (ITN STRONGnet). Critical to this calculation were the Blue Gene/Q computers at the Argonne Leadership Computing Facility (DOE contract DEAC02-06CH11357) as well as the DOE USQCD and RIKEN BNL Research Center Blue Gene/Q computers at the Brookhaven National Lab. NHC was supported in part by US DOE grant DEFG02-92ER40699.

\section{References}

[1] G. Colangelo, S. Dürr, A. Jüttner, et al. Review of lattice results concerning low-energy particle physics. The European Physical Journal C, 71(7):1-76, 2011.

[2] Jack Laiho, E. Lunghi, and Ruth S. Van de Water. Lattice qcd inputs to the ckm unitarity triangle analysis. Phys. Rev. D, 81:034503, Feb 2010.

[3] Y. Aoki, R. Arthur, T. Blum, et al. Continuum limit of $B_{K}$ from $2+1$ flavor domain wall qcd. Phys. Rev. D, 84:014503, Jul 2011.

[4] R. Arthur, T. Blum, P. A. Boyle, et al. Domain wall qcd with near-physical pions. Phys. Rev. D, 87:094514, May 2013.

[5] Thomas Blum, Taku Izubuchi, and Eigo Shintani. A new class of variance reduction techniques using lattice symmetries. 2012, arXiv:1208.4349.

[6] Damir Becirevic and Giovanni Villadoro. Impact of the finite volume effects on the chiral behavior of $\mathrm{f}(\mathrm{K})$ and B(K). Phys.Rev., D69:054010, 2004, hep-lat/0311028. 